\title{
Correction: Rapamycin increases CCN2 expression of lung fibroblasts via phosphoinositide 3-kinase
}

\author{
Xuefeng Xu $\cdot$ Huaping Dai $\cdot$ Jing Geng $\cdot$ Xuan Wan $\cdot$ Xiaoxi Huang $\cdot$ Fei Li $\cdot$ Dianhua Jiang $\cdot$ Chen Wang
}

(c) USCAP, inc All Rights Reserved 2020, corrected publication 2021

Correction to: Laboratory Investigation

https://doi.org/10.1038/labinvest.2015.68

Following the publication of the article listed above, a reader noted that Figs. 8A and 9A contained same p-AKT gel images. The authors have indicated that the errors occurred during figure preparation. We have provided the corrected panel below. Although these errors do not affect the conclusion of the article, the authors deeply regret the mistakes. 
A
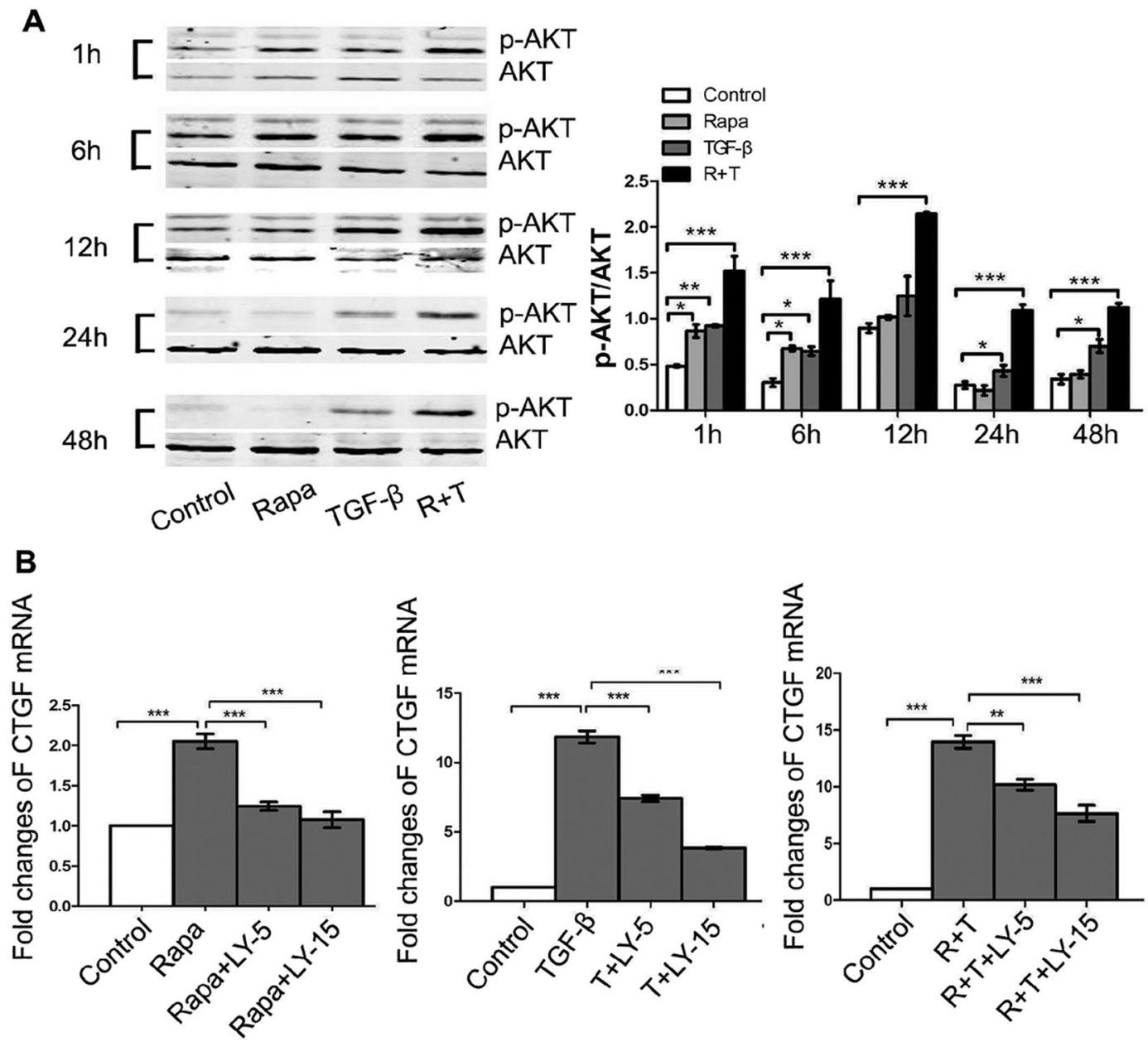

Corrected Fig. 8 PI3K-AKT was involved in CCN2 expression of normal fibroblast. (a) Serum-starved N-HLFs were stimulated with either vehicle (-) or rapamycin $(5 \mathrm{ng} / \mathrm{ml})$, TGF- $\beta 1(10 \mathrm{ng} / \mathrm{ml})$ or both and were lysed for western blot analysis. Left panel: The typical gel is shown to represent three independent experiments (each with duplicates) from three different cell lines giving similar results. Molecular weight: (p)AKT, $56 \mathrm{KDa}$. Right panel: Densitometric analysis as the ratio of p-AKT to total AKT is shown with mean \pm s.e.m. $(n=3, * P<0.05$, $* * P<0.01$, $* * * P<0.001$ vs. control group). (b) After incubations with rapamycin, TGF- $\beta 1$, or both in the presence of LY-294002 for $24 \mathrm{~h}$, CCN2 mRNA steady-state levels were determined by quantitative RT-PCR. Bars indicate mean \pm s.e.m. of three independent experiments performed, each with duplicates. $* *, * * *$ Significant difference, $P<0.01, P<0.001$, respectively. 